\title{
Polyacrylamide Hydrogel
}

National Cancer Institute

\section{Source}

National Cancer Institute. Polyacrylamide Hydrogel. NCI Thesaurus. Code $C 95765$.

A transparent, biocompatible, non-resorbable, homogenous hydrogel containing $97.5 \%$ apyrogenic water and $2.5 \%$ of the cross-linked, synthetic polyacrylamide. Upon injection into the urethral submucosal tissue, the polyacrylamide hydrogel serves as a bulk forming agent and may possibly increase the strength of the urethral sphincter and thereby prevent or decrease stress-induced urinary incontinence. 